\title{
Lumen
}

Selected Proceedings from the Canadian Society for Eighteenth-Century Studies

\section{Les Lettres persanes ou l'exotisme sans l'exotisme}

\section{Pierre Berthiaume}

Volume 24, 2005

Indigenes and Exoticism

Indigènes et exotisme

URI : https://id.erudit.org/iderudit/1012171ar

DOI : https://doi.org/10.7202/1012171ar

Aller au sommaire du numéro

Éditeur(s)

Canadian Society for Eighteenth-Century Studies / Société canadienne d'étude du dix-huitième siècle

ISSN

1209-3696 (imprimé)

1927-8284 (numérique)

Découvrir la revue

Citer cet article

Berthiaume, P. (2005). Les Lettres persanes ou l'exotisme sans l'exotisme.

Lumen, 24, 1-18. https://doi.org/10.7202/1012171ar

Copyright (C) Canadian Society for Eighteenth-Century Studies / Sociéte canadienne d'étude du dix-huitième siècle, 2005
Ce document est protégé par la loi sur le droit d'auteur. L'utilisation des services d'Érudit (y compris la reproduction) est assujettie à sa politique d'utilisation que vous pouvez consulter en ligne.

https://apropos.erudit.org/fr/usagers/politique-dutilisation/ 


\section{Les Lettres persanes ou l'exotisme sans l'exotisme}

Les écrivains du siècle des Lumières, on le sait, n'ont guère cultivé l'exotisme. Que ce fût à cause de leurs convictions rationalistes ou de leur insensibilité à l'endroit d'une nature qui ne parle pas leur langage d'ordre et de civilité ${ }^{1}$, les contemporains de Voltaire ont davantage cherché à asseoir l'universalité du genre humain qu'à exalter son étrangeté. Aussi, point $\mathrm{d}^{\prime}$ «expérience existentielle ${ }^{2}$ » chez eux, qui les eût poussés à "devenir excessivement chinois", pour reprendre une image de Victor Segalen ${ }^{3}$. En outre, rappelle Denise Brahimi, l'idéologie scientifique "paraît incompatible avec l'exotisme en ce qu'elle refuse l'inconnaissable, au moins en droit, et ne lui laisse aucune place ${ }^{4} »$. Explorateurs, missionnaires et voyageurs entendaient faire connaître le monde, plutôt que faire rêver sur lui. Pour tout dire, on a le sentiment que le $\mathrm{XVIII}{ }^{\mathrm{e}}$ siècle arrive trop tard ou trop tôt. Trop tard par rapport au $\mathrm{XVI}^{\mathrm{e}}$ siècle, qui donne naissance à l'épithète «exotique ${ }^{5}$ »;

1 Voir Michèle Duchet, "Aspects de la littérature française de voyage au XVIII ${ }^{e}$, Cahiers $d u$ Sud, 1966, no 389, p. 12. A la différence d'un Pierre Loti, par exemple, les contemporains de Voltaire ne sont guère sensibles à la bizarrerie d'un décor «qu'on eût dit moins une réalité qu'une composition fantastique de quelque orientaliste halluciné» (Aziyadé, dans Aziyadé suivi de Fantôme d'Orient, édition de Claude Martin, Paris, Gallimard, «Folio classique», 1991, p.76).

2 Expression empruntée à D. Brahimi, «Exotisme, science et idéologie», Studies on Voltaire and the Eighteenth Century, 1976, vol. 151, p. 376-377.

3 V. Segalen, Essai sur l'exotisme. Une esthétique du divers (notes), Paris, Fata Morgana, 1978, p. 48.

4 D. Brahimi, article cité, p. 371. Selon Dick Van der Cruysse, l'exotisme prend parfois la forme du lyrisme à l'endroit de l'altérité: voir «L'exotisme pluriel de Raynal: le discours exotique dans l'“Histoire des deux Indes"», French Literature Series, 1986, vol. XIII, p. 13-27.

5 Alors qu'il explore le havre de l'île Medamothi, qui est aussi grande que le Canada, Pantagruel voit «divers tableaux, diverses tapisseries, divers animaulx, poissons, oizeaulx et aultres marchandises exotiques et pérégrines, qui estoient en l'allée du 
trop tôt pour le substantif «exotisme», qui n'apparaît qu'au siècle suivant ${ }^{6}$.

L'exotisme, observe Victor Segalen, se développe alors selon deux axes: il peut se fonder sur les «impressions» du voyageur, dont les expériences et les aventures forment la trame du récit, ou, au contraire, illustrer la «réaction non plus du milieu sur le voyageur, mais du voyageur sur le milieu", comme dans Les Immémoriaux, où la venue de missionnaires occidentaux est racontée selon le point de vue des Maoris ${ }^{7}$.

Les Lettres persanes, avec leur chassé-croisé entre une Perse mystérieuse aux Français et une Europe étrange aux yeux des Persans ${ }^{8}$, se prêtaient à l'exploitation de l'exotisme et pouvaient même s'inscrire

môle et par les halles du port» (François Rabelais, Le Quart Livre, 1548, dans Oeuvres complètes, Paris, Gallimard, Bibliothèque de La Pléiade, 1955, p. 543). Voir Roger Mathé, L'Exotisme d'Homère à Le Clézio, Paris, Bordas, "Univers des Lettres», 1972, p. 13. L'adjectif ne fera son entrée dans le Dictionnaire de l'Académie française qu'en 1765 (voir Vincenette Maigne, «Exotisme: évolution en diachronie du mot et de son champ sémantique», dans Exotisme et création. Actes du Colloque international de Lyon, préface de Roland Antonioli, Lyon, L'Hermès, 1985, p. 7).

6 Voir infra, p. 18.

7 V. Segalen, op. cit., p. 17-18. Dans «Enjeux et risques du roman exotique français», Denise Brahimi a repris la même distinction entre "Aventure du voyageur", où les émotions du voyageur forment l'essentiel du récit, et "Les Autres parlent», où "la fiction romanesque entend décrire, évoquer, faire vivre et parler le monde de l'autre, comme s'il était autonome et parlait de lui-même pour lui-même» (dans Alain Buisine, Norbert Dodille et Claude Duchet (sous la direction de), L'Exotisme, La Réunion, Faculté des lettres et sciences humaines, Cahiers CRLH-CIRAOI, ${ }^{\circ}$ 5, 1988, p. 12). Dans la première catégorie, «c'est le pôle européen qui l'emporte, par exemple les émotions, sentiments, aventures du sujet européen, aux dépens de l'autre pôle, qui peut être très abondamment décrit, mais non pour lui-même» (ibid., p. 12). Ainsi, «tout doit y être représenté du point de vue de l'Européen, qui ignore l'essentiel du mode de vie, des rapports et des sentiments en vigueur dans cette société étrangère» (ibid., p. 14). Dans la seconde catégorie, elle produit l'exemple des Immémoriaux, de Victor Segalen, ce dernier s'étant employé à narrer l'arrivée des missionnaires en Océanie «du point de vue des Maoris, conformément à leur mentalité, à leur système de valeurs, en tenant compte de leur effarement devant les valeurs étrangères, puis de leur adaptation singulière au nouvel état de choses» (ibid., p. 15).

8 Pour preuve, le recours aux adjectifs «bizarres» (Montesquieu, Lettres persanes. Texte établi, avec introduction, chronologie de Montesquieu, biographie, notes et relevé de variantes par Paul Vernière, Paris, Éditions Garnier Frères, 1960, p. 104, 157, 220, 294), «étonnant» (p. 205), «extraordinaire» (p. 215) et «singulier» (p. 53, 63, 92, 157, 173, 220, 273), auxquels on peut joindre les verbes «admirer» (p. 78, 158, 295, 317, 334) et «étonner» (p. 55, 69, 98, 131). A l'avenir, nous nous contenterons d'indiquer L. P., suivi de la page. 
dans les deux catégories définies par Victor Segalen. D'une part, Usbek et Rica découvrent l'Europe et leurs lettres traduisent leur effarement devant les mœurs singulières des Européens, en même temps qu'elles rendent compte des incidents de leur séjour; d'autre part, les deux Persans attestent les valeurs de leur société d'origine, ce dont témoignent la "poésie monacale sur le harem», la "couleur orientale», la "science du harem» de certaines lettres, voire les scènes de sérail inspirées des Voyages de Chardin et de Tavernier?.

Mais si persans, ou plutôt perçants, que soient les yeux des deux voyageurs orientaux ou de leurs proches, leurs lettres demeurent singulièrement dénuées d'exotisme. Alors qu'il vient à peine de poser le pied sur les quais de Livourne, en Toscane, Usbek avoue à Ibben: «il y a jusque dans les moindres bagatelles, quelque chose de singulier que je sens, et que je ne sais pas dire ${ }^{10}$ ». Jamais les Persans ne se préoccupent de dégager «le beau du bizarre» afin de conférer une valeur esthétique ou émotive à ce qu'ils voient ${ }^{11}$. Leurs lettres ne stimulent que fort peu l'imagination du lecteur.

Et pour cause. Peut-on concevoir l'exotisme sans le recours aux sens? Chez Eugène Fromentin, comme chez Pierre Loti ou Pierre Benoît, non seulement la vue, mais l'odorat, l'ouie, le tact, le goût sont mis à contribution, qui ancrent le lecteur dans un univers lointain, différent du sien. Semblables à Vandell et au narrateur d'Une année dans le Sahel, les lecteurs sont «des sensuels, agréablement parfumés par le voisinage d'une plante exotique $^{12}{ }^{2}$. A travers les sens, l'exotisme s'immisce dans le lecteur, l'imprègne tout entier, partant, se confond avec son vécu intime. Mais dans les Lettres persanes, le seul sens qui a droit de cité est la vue, c'est-à-dire un sens qui maintient une distance entre les choses et les êtres. Impossible, dès lors, d'être en correspondance avec l'étrangeté de l'ailleurs, qu'il soit persan ou français. Avec la vue, seule demeure

9 Voir les notes de Paul Vernière, L. P., p. 13, n. 1, p. 39, n. 4, p. 43, n. 2, p. 48, n. 2, etc.

10 L. P., p. 53.

11 Eugène Fromentin, Une année dans le Sahel, Paris, Gf-Flammarion, 1991 (1859), p. 186. Pour le peintre, il s'agit essentiellement de donner accès à des lieux inconnus au public européen, de lui «faire admettre les plus périlleuses nouveautés par des moyens d'expression usuels», de faire en sorte que ce qui est insolite "devienne un tableau sensible, intelligible et vraisemblable» (ibid., p. 186). En même temps, l'imaginaire et le rêve jouent un rôle essentiel dans l'élaboration de l'exotisme et Eugène Fromentin qualifie le toponyme Afrique de «mot magique, qui prête aux conjectures, et qui fait rêver les amateurs de découvertes» (ibid., p. 38).

12 E. Fromentin, op. cit., p. 150. 
l'esprit de géométrie qui examine les choses, plutôt qu'il ne les laisse envahir les êtres.

L'appel de l'ailleurs, le désir de faire la découverte de ce qui est différent, insolite, et qui repose sur une vacance de l'être, sur une attente, est aussi totalement absent des Lettres persanes. Dans les récits exotiques, l'expérience de l'ailleurs forme un hors-temps, un vide que remplissent des émotions: émerveillement devant la splendeur du désert chez Fromentin, envoûtement sensuel et amoureux chez Loti. Une illumination surgit, qui déstabilise l'être et qui met à l'épreuve sa vie ${ }^{13}$. Le contact avec l'altérité dévoile alors des recoins obscurs, inconnus de l'être. Or, même s'ils sont partis de Perse «pour aller chercher laborieusement la sagesse ${ }^{14}{ }$, Rica et Usbek ne sont jamais marqués par la culture occidentale. Leur regard demeure critique, distant. Ni l'un, ni l'autre ne laissent entrevoir quelque émotion que ce soit, ni nostalgie à l'endroit du pays quitté, ni bonheur de la découverte de l'Europe. Dans les Lettres persanes, l'ailleurs n'est ni l'objet d'un désir, ni l'occasion d'un mirage.

Point d'exotisme européen. Point d'exotisme oriental, non plus. Au mieux, quelques scènes de sérail peuvent-elles faire rêver un lecteur français. Ainsi l'évocation de la «querelle entre les femmes» au sujet de leur beauté, qui les amène à défiler devant Usbek, d'abord couvertes «de parures et d'ornements», puis "dans la simplicité de la nature», jusqu'à $1^{\prime}$ amener à «errer d'enchantements en enchantements ${ }^{15}$ », ne manque-t-elle pas de couleur locale. Mais la scène, si saturée soit-elle de sensualité, n'est pas sans rappeler le jugement de Céladon dans Astrée $^{16}$, sinon celui de Pâris. Il s'agit moins d'exotisme que de souvenir de collège ${ }^{17}$.

Sur le plan terminologique, le constat n'est guère plus encourageant. Malgré l'artifice d'une chronologie musulmane en surimpression sur les

13 Qu'on pense à la fièvre qui parcourt L'Atlantide de Pierre Benoît et qui donne au récit de Ferrières une dimension presque mythique.

14 L. P., p. 12. Sur cette quête et la mise en doute de «l'idéalisme du savoir», voir Sylvie Romanowski, "La quête du savoir dans les Lettres persanes», Eighteenth-Century Fiction, janvier 1991, vol. 3, no 2, p. 93-111.

15 L. P., p. 15.

16 Voir Honoré d'Urfé, L'Astrée. Nouvelle édition publiée sous les auspices de la «Diana» par M. Hugues Vaganay, Genève, Slatkine Reprints, 1966 (1925-1928), Première partie, livre quatrième, t. I, p. 115-116.

17 Il est vrai, comme le rappelle Gilbert Chinard, qu'au XVII ${ }^{\mathrm{e}}$ et au XVIII ${ }^{\mathrm{e}}$ siècle l'altérité était perçue à travers les «souvenirs de l'Antiquité» (L'Amérique et le rêve exotique dans la littérature au XVII et XVIII siècle, Genève, Slatkine Reprints, 1970 (1913), p. VI). 
mois du calendrier grégorien ${ }^{18}$ et le recours à des termes qui évoquent un monde lointain, le roman de Montesquieu n'entraîne pas le lecteur dans un univers différent du sien. Sur le plan stylistique, à peine trouvet-on dans les Lettres persanes quelques passages au style oriental. Par exemple, la lettre du mollah "Méhémet-Hali, serviteur des prophètes», avec ses termes persans et ses images grandiloquentes, peut sembler exotique:

Profanes, qui n'entrez jamais dans les secrets de l'Éternel, vos lumières ressemblent aux ténèbres de l'Abîme, et les raisonnements de votre esprit sont comme la poussière que vos pieds font élever lorsque le Soleil est dans son midi, dans le mois ardent de Chahban. Aussi le zénith de votre esprit ne va pas au nadir de celui du moindre immaums. Votre vaine philosophie est cet éclair qui annonce l'orage et l'obscurité; vous êtes au milieu de la tempête, et vous errez au gré des vents ${ }^{19}$.

Bel exemple, que suit Usbek lorsqu'il écrit au mollah:

Que nous servent les jeûnes des immaums et les cilices des mollaks ? La main de Dieu s'est deux fois appesantie sur les enfants de la Loi: le Soleil s'obscurcit et semble n'éclairer plus que leurs défaites; leurs armées s'assemblent, et elles sont dissipées comme la poussière ${ }^{20}$.

Mais ces morceaux de rhétorique persane, fondée sur l'accumulation de comparaisons, de métaphores et d'antithèses, assez peu nombreux, du reste, forment au mieux des balises qui, placées à intervalle plus ou moins régulier dans l'oeuvre, ont pour fonction d'entretenir la fiction romanesque. D'ailleurs, la "couleur orientale» de la lettre, inspirée du poète persan Al-Hasan ben al-Khasib ${ }^{21}$, se délave rapidement: la lettre suivante, écrite par Usbek et adressée au mollah, ne comporte plus rien d'exotique, Usbek interrogeant Méhémet-Hali sur des questions religieuses. La réflexion philosophique passe avant la couleur locale. Au

18 Voir Robert Shackleton, «The Moslem Chronology of the Lettres persanes», French Studies, janvier 1954, p. 17-27; Paul Vernière, «Introduction», dans Montesquieu, L. P., p. XI-XIII.

19 L. P., p. 43. Voir les commentaires de P. Vernière (p. 43, notes 1, 2 et 3 ).

20 L. P., p. 260.

21 Sur cette poésie de mille et une nuits françaises, voir P. Vernière, dans L. P., p. 39-40, n. 4 . 
vrai, les Persans de Montesquieu écrivent le plus souvent comme des Français et l'histoire des Troglodytes, rapportée par Usbek, ou celle d'Aphéridon et d'Astarté, racontée par Ibben, ne diffèrent guère sur le plan stylistique du "conte persan", qui, traduit en français par une dame de la Cour, aurait été «travesti», au dire de Rica ${ }^{22}$.

À défaut de style oriental qui risquait de rebuter le lecteur, Montesquieu aurait pu produire des descriptions qui eussent mis l'accent sur les singularités de pays lointains et peu connus. D'ailleurs, la description, on le sait, constitue le fonds de commerce de l'exotisme ${ }^{23}$. Malheureusement, les Persans paraissent peu enclins à décrire ce qu'ils voient. Ainsi, lorsqu'elle rappelle les événements qui ont suivi sa réconciliation avec Zéphis, le "grand festin» où furent invitées la mère d'Usbek, ses femmes, ses principales concubines, ses tantes et plusieurs de ses cousines «venues à cheval, couvertes du sombre nuage de leurs voiles et de leurs habits», Zachi fait-elle l'économie de la description du repas ${ }^{24}$. Son récit du voyage à la campagne, le lendemain, est à peine plus développé:

Nous montâmes sur nos chameaux, [raconte-t-elle,] et nous nous mîmes quatre dans chaque loge. Comme la partie avait été faite brusquement, nous n'eûmes pas le temps d'envoyer à la ronde annoncer le courouc; mais le premier Eunuque, toujours industrieux, prit une autre précaution: car il joignit à la toile qui nous empêchait d'être vues un rideau si épais que nous ne pouvions absolument voir personne $e^{25}$.

Le lecteur ne voit guère mieux. Si, au début du récit, il peut imaginer sans trop de peine la caravane des bêtes, bâtées de berceaux fermés dans lesquels ont pris place les femmes, il imagine moins facilement la fâcheuse rencontre d'un curieux qui «reçut un coup mortel, qui lui ôta pour jamais la lumière du jour» ou celle d'un homme qui «eut le même sort» pour avoir été surpris en train de se baigner nu. Le récit fait

22 L. P., p. 296. Il est vrai que dans son «Introduction», Montesquieu précise que toute sa "peine a été de mettre l'ouvrage à nos moeurs» et qu'il a «soulagé le lecteur du langage asiatique» autant qu'il a pu (L. P., p. 7-8).

23 Parmi les procédés littéraires caractéristiques de l'exotisme, Roger Mathé signale le recours à l'image qui «s'organise en tableaux» (op. cit., p. 25). Mais avant Bernardin de Saint-Pierre, on ne trouve guère de «tableaux».

24 L.P., p. 96.

25 L. P., p. 96-97. 
l'économie du décor, tout autant que des événements, dont il n'expose que les résultats ${ }^{26}$.

Plus bas, lorsque le chef des eunuques noirs rappelle son arrivée dans le sérail de son premier maître, il observe qu'il découvrit alors «un nouveau monde». Pourtant, la description qu'il fait du lieu manque singulièrement de couleurs:

On n'y entendait parler ni de divisions ni de querelles: un silence profond régnait partout; toutes ces femmes étaient couchées à la même heure, d'un bout de l'année à l'autre, et levées à la même heure; elles entraient dans le bain tour à tour; elles en sortaient au moindre signe que nous leur faisions; le reste du temps, elles étaient presque toujours enfermées dans leurs chambres ${ }^{27}$.

Rien de bien pittoresque dans ces lignes, marquées par l'absence de précisions sur les bains, sur la décoration des chambres ou sur l'habillement des femmes ${ }^{28}$. Il est vrai que l'exotisme de la lettre ne peut faire que long feu, le chef des eunuques ne pouvant décrire sous un angle exotique ce que son destinataire connaît aussi bien que lui. Ne fût-ce que pour maintenir la fiction épistolaire des échanges entre personnages issus d'une même culture orientale, Montesquieu devait s'interdire presque toute forme d'exotisme. Aussi la description se réduit-elle à un exposé sur l'organisation des activités quotidiennes.

C'est aussi pour des raisons de vraisemblance littéraire que Zachi, au début du roman, raconte moins les péripéties de son voyage à la campagne avec les autres femmes du sérail qu'elle n'insiste sur sa détresse amoureuse. Elle évoque l'événement en quelques lignes ${ }^{29}$ pour mieux

26 D'ailleurs, lorsque les voyageuses sont menacées par les flots que soulève un vent impétueux, elles perdent opportunément connaissance et la narratrice ne revient à elle «qu'après que le péril fut passé» (L. P., p. 98).

27 L.P., p. 133.

28 Mais peut-être la description n'était-elle pas sans un certain charme pour un lecteur d'autrefois, moins contaminé que nous par l'iconographie télévisuelle et cinématographique.

29 «Nous avons ordonné au chef des eunuques de nous mener à la campagne; il te dira qu'aucun accident ne nous est arrivé [écrit-elle à Usbek]. Quand il a fallu traverser la rivière et quitter nos litières, nous nous mîmes, selon la coutume, dans des boîtes: deux esclaves nous portèrent sur leurs épaules, et nous échappâmes à tous les regards» (L. P., p. 14). Elle ne décrit ni le cadre géographique dans lequel évolue la petite troupe, ni celle-ci. Tout cela, Usbek le connaît en principe. 
laisser libre cours à ses plaintes et à ses doléances ${ }^{30}$. Tout aussi émotive, Fatmé, qui ne se "couche point» qu'elle ne se soit "parfumée des essences les plus délicieuses", et qui suggère par là des moeurs sans doute quelque peu insolites aux yeux des contemporains de Montesquieu, insiste moins sur le rituel qui précède son coucher qu'elle ne souligne son désarroi sentimental, elle dont $\mathrm{l}^{\prime}$ «imagination se perd dans ses désirs, comme elle se flatte dans ses espérances ${ }^{31}{ }^{\prime}$. L'expression $d u$ sentiment prend le pas sur la description; l'exotisme est oblitéré par une émotion qui n'a rien de singulier et le roman se fait lyrique:

Que ne puis-je t'exprimer ce que je sens si bien [écrit-elle à Usbek] et comment sens-je si bien ce que je ne puis t'exprimer! Dans ces moments, Usbek, je donnerais l'empire du Monde pour un seul de tes baisers. Qu'une femme est malheureuse d'avoir des désirs si violents, lorsqu'elle est privée de celui qui peut seul les satisfaire ${ }^{32}$.

Foin de l'exotisme persan ou européen dans ces lettres qui mettent davantage l'accent sur l'expression des émotions des personnages, ou sur leurs réflexions, plutôt que sur ce qu'ils voient. Si «surpris» et si «étonné» qu'il fût en arrivant dans la cité des doges «de voir une ville, des tours et des mosquées sortir de dessous l'eau, et de trouver un peuple innombrable dans un endroit où il ne devrait y avoir que des poissons ${ }^{33}$ ", Rhédi n'insiste toutefois pas sur les caractéristiques architecturales de la cité lacustre, trop pressé qu'il est de dénoncer l'impossibilité «d'y accomplir une seule ablution légale». La description cède au jugement: Venise «est en abomination à notre saint Prophète; il ne la regarde jamais du haut du Ciel, qu'avec colère», conclut le voyageur ${ }^{34}$.

$\mathrm{Du}$ reste, à cause des rapports entre les personnages, les lettres décrivent moins les événements qu'elles ne présentent leur vécu de façon synthétique et sous un angle explicatif. Par exemple, au lieu de donner à voir ses activités au sein du sérail lorsqu'il écrit à Ibbi, le premier eunuque en rend compte de façon générale:

30 Avant, il est vrai, d'évoquer la «querelle» sur la beauté des femmes, dont nous avons parlé plus haut.

31 L. P., p. 20.

32 L.P.,p. 20. Le rythme de la phrase, les inversions, les exclamatives appartiennent plus au théâtre qu'au récit exotique.

33 L.P., p. 69.

34 L.P., p. 70. 
Je m'arme de refus; je me hérisse de scrupules; je n'ai jamais dans la bouche que les mots de devoir, de vertu, de pudeur, de modestie. Je les désespère en leur parlant sans cesse de la faiblesse de leur sexe et de l'autorité du maitre. Je me plains ensuite d'être obligé à tant de sévérité et je semble vouloir leur faire entendre quejen'ai d'autre motif que leur propre intérêt et un grand attachement pour elles ${ }^{35}$.

Il dresse le bilan de son activité; il en fait la synthèse sans l'illustrer. Lorsqu'il explique ensuite que les femmes du sérail lui rendent la monnaie de sa pièce et lui font la vie dure, il a recours au même procédé:

Elles ont des revers terribles. Il y a entre nous comme un flux et un reflux d'empire et de soumission. Elles font toujours tomber sur moi les emplois les plus humiliants; elles affectent un mépris qui n'a point d'exemple; et, sans égard pour ma vieillesse, elles me font lever la nuit dix fois pour la moindre bagatelle ${ }^{36}$.

Il ne précise pas les emplois humiliants auxquels il fait allusion, pas plus qu'il n'illustre le mépris dont il est l'objet ou qu'il ne met en scène ses levers, la nuit. Il se contente de recenser ses maux, sans jamais les décrire précisément.

Dans les Lettres persanes, les événements et les choses sont rarement présentés dans leur singularité, dans leur nudité. A cause de la forme épistolaire du roman, événements et vécu intime sont médiatisés. Entre l'expérience prêtée aux personnages et leur témoignage sur celle-ci, s'interpose la lettre, qui se fonde sur un échange intellectuel, si bien que l'exotisme passe au second plan $^{37}$ :

Quand vous relevez l'éclat de votre teint par les plus belles couleurs; quand vous vous parfumez tout le corps des essences les plus précieuses; quand vous vous parez de vos plus beaux habits; quand vous cherchez à vous distinguer de vos compagnes par les grâces de la danse et par la douceur de votre chant; que vous

35 L.P., p. 25.

36 L.P., p. 25.

37 Dans les récits exotiques, les narrateurs exposent plutôt leur expérience sous forme de journal ou de récit continu qui témoignent d'une forme d'intimité entre l'expérience vécue et le récit qu'ils en font. Aucune ou très peu de distance critique entre l'événement et le récit, même chez Fromentin, qui rapporte son expérience algérienne dans des lettres, qui tissent finalement la trame d'un récit continu. 
combattez gracieusement avec elles de charmes, de douceur et d'enjouement; je ne puis m'imaginer que vous ayez d'autre objet que celui de me plaire ${ }^{38}$,

écrit Usbek, qui décrit moins les gestes de Roxane qu'il n'analyse le sens de ceux-ci. La description n'a d'autre fonction que de préluder à une réflexion.

L'intellectualisation constante dans le roman conduit les personnages à énoncer des principes, plutôt qu'à décrire. Ainsi, Usbek, au lieu d'illustrer l'activité de son eunuque, définit-il ses tâches presque sous forme de consignes. Il expose alors les principes sur lesquels repose l'organisation d'un sérail, beaucoup plus qu'il ne donne à voir son fonctionnement:

Tu es le gardien fidèle des plus belles femmes de Perse; je t'ai confié ce quej'avais dans le Monde de plus cher; tu tiens en tes mains les clefs de ces portes fatales qui ne s'ouvrent que pour toi. Tandis que tu veilles sur ce dépôt précieux de mon coeur, il se repose et jouit d'une sécurité entière. Tu fais la garde dans le silence de la nuit, comme dans le tumulte du jour; tes soins infatigables soutiennent la vertu lorsqu'elle chancelle ${ }^{39}$.

Le texte se poursuit sur une série d'injonctions qui énumèrent plus les règles d'un sérail qu'elles ne décrivent précisément l'activité des eunuques ou celle des femmes:

Tiens-toi dans un profond abaissement auprès de celles qui partagent mon amour; mais fais-leur en même temps sentir leur extrême dépendance. Procureleur tous les plaisirs qui peuvent être innocents; trompe leurs inquiétudes; amuse-les par la musique, les danses, les boissons délicieuses; persuade-leur de $\mathrm{s}^{\prime}$ assembler souvent ${ }^{40}$.

Le caractère intellectuel des Lettres persanes, qui tient de l'esprit des Lumières, sape en grande partie l'exotisme que peuvent contenir ces lignes.

Pour preuve, la description de la Turquie par Usbek, ou plutôt l'absence de description du pays. Bien qu'il séjourne «trois ou quatre mois ${ }^{41}$ » à Erzeron (Erivan) et un peu plus de deux mois à Smyrne, et qu'il y écrive

38 L. P., p. 60-61.

39 L. P., p. 12-13.

40 L.P., p. 13.

41 L. P., p. 17. 
douze lettres ${ }^{42}$, Usbek ne produit aucune description du pays. En revanche, il dresse un bilan analytique de l'empire des Osmanlins et décrie un «corps malade» qui ne se soutient que "par des remèdes violents, qui l'épuisent et le minent sans cesse ${ }^{43}{ }^{\prime}$. L'analyse prend le pas sur la description. Au lieu de regarder en voyageur, Usbek réfléchit en philosophe, voire en sociologue. Les rares descriptions, par exemple celle qui met en scène une jeune femme qui doit faire le pied de grue à la porte de la maison de son futur époux parce que ce dernier exige qu'on majore sa dot et qui est renvoyée à son père sous prétexte qu'elle n'est pas vierge ${ }^{44}$, permet surtout de dénoncer une injustice qu'autorise la loi musulmane ${ }^{45}$. Lorsque Rica rencontre une «dame de la Cour qui avait quelque envie de voir [sa] figure étrangère» et qui lui pose «mille questions sur les moeurs des Persans, et sur la manière de vivre des Persanes", il n'évoque pas les coutumes de son pays, mais signale plutôt la "répugnance» de son interlocutrice à l'endroit de la polygamie pratiquée en Perse ${ }^{46}$.

À lire les Lettres persanes, on a le sentiment que Montesquieu s'est évertué à gommer tout exotisme de son roman. Usbek, qui est devenu "plus métaphysicien» qu'il ne l'était au moment de quitter Ispahan, n'a de cesse de faire essuyer à ses lecteurs le «débordement de [sa] philosophie $^{47}$ ». Or rien de moins exotique que la philosophie. Quant à Rica, ses observations sur la société européenne, qui rappellent parfois celles d'un La Bruyère, relèvent d'un moralisme teinté $d$ 'ironie. Chez Montesquieu, le philosophe et le moraliste tiennent la bride au romancier.

Pourtant, dans les Lettres persanes, Montesquieu définit les conditions d'existence de l'exotisme, en même temps qu'il en illustre l'usage. Mais ce n'est pas dans la facture du récit qu'il faut cherche l'exotisme; c'est dans le mode d'approche de l'altérité, de l'Autre.

Les choses et les êtres ne sont pas exotiques en soi. C'est le regard qui confère aux êtres et aux choses une dimension exotique en les super-

42 Lettres VI (à Nessir), VIII, XIX (à Rustan), XI, XII, XIII, XVIV (à Mirza), XVI, XVII, XVIII (à Méhémet-Hali), XX (à Zachi), XXI (à son eunuque blanc).

43 L. P., p. 46. La lettre met l'accent sur les faiblesses de la Turquie, sans jamais décrire précisément sa situation: voir p. 46-48.

44 L. P., lettre LXX, p. 153-154.

45 Voir L. P., lettre LXXI, p. 154-155.

46 L. P., p. 296.

47 L.P., p. 149. 
posant sur ce qui est connu et convenu, en superposant le différent sur le même. L'exotisme est une construction de l'esprit fondé sur un jeu d'échange entre un ici et un ailleurs. Comme l'observe Jean-Michel Racault:

L'Autre ne saurait être tel que par référence au Même; on ne peut l'appréhender que dans une relation duelle dont le monde de départ constitue l'autre terme. [...] l'altérité apparaît bien comme un concept différentiel, ou mieux relationnel. [...] C'est dire que la perspective exotique n'appelle pas un simple transfert du regard dans un espace autre, mais la mise en relation de deux espaces, la présence en arrière-plan d'un monde de référence identifié à la norme, celle de l'auteur et du lecteur ${ }^{48}$.

C'est ce «monde de référence» qui rend possible l'exotisme. Dans son analyse du substantif, Vincenette Maigne observe que lorsque le champ sémantique du terme s'élargit et passe des productions qui ne sont pas indigènes au domaine culturel, le "glissement s'accompagne du passage d'une valeur purement objective ('d'ailleurs, étranger') à une valeur impressive ('étrange' $)^{49}{ }{ }$. Cette évolution ne peut s'opérer qu'au moment où l'on pense le monde à partir d'un point de vue qui confère à sa culture, à sa civilisation valeur de norme. N'est étrange, n'est exotique que ce qui se distingue de la norme. Aussi, l'exotisme, au sens d'étrangeté, ne peut-il apparaître qu'au moment où l'Europe postule l'universalité de la condition humaine. La bigarrure des êtres humains, qui constituait l'essence de l'humanité aux yeux d'un Montaigne, se réduit, au siècle de la raison, à des marques secondaires. Dire d'autrui qu'il est exotique, c'est à la fois reconnaître son appartenance au fonds commun humain et souligner un écart. Lorsque Rica annonce à Ibben

48 Jean-Michel Racault, «Instances médiatrices et production de l'altérité dans le récit exotique aux $17^{\mathrm{e}}$ et $18^{\mathrm{e}}$ siècles", dans Alain Buisine, Norbert Dodille et Claude Duchet (sous la direction de), op. cit., p. 33. Le texte de l'article est repris dans Nulle part et ses environs. Voyage aux confins de l'utopie littéraire classique (1657-1802), Paris, Presses de 1'Université de Paris-Sorbonne, 2003, p. 357-369. Pour Jean-Michel Racault, «le tableau de l'ailleurs a pour vocation de faire retour à l'ici en une confrontation qui lui donne son sens" (ibid., p. 34). Aussi, à ses yeux, l'exotisme, au XVIII ${ }^{\mathrm{e}}$ siècle est-il soumis à un projet utopique, l'ailleurs étant investi «d'une signification critique, exemplaire ou simplement compensatoire face à une réalité socio-politique contestée» (ibid., p. 33). Rappelons que le problème posé par «l'interprétation» de l'ailleurs, notamment de l'Orient, est aussi étudié par Eugène Fromentin (voir Une année dans le Sahel, p. 184-187).

Vincenette Maigne, article cité, p. 10. 
qu'il lui apprendra «des choses bien éloignées du caractère et du génie persan», il s'empresse d'ajouter: «c'est bien la même Terre qui nous porte tous deux; mais les hommes du pays où je vis, et ceux du pays où tu es, sont des hommes bien différents ${ }^{50}$ ». L'universalité de la condition humaine constitue la toile de fond sur laquelle peut se profiler l'exotisme ${ }^{51}$.

Aussi, avant d'être une reconnaissance de la différence de l'Autre, l'exotisme est-il une forme d'assimilation de celui-ci. Tel un code syntaxique, le regard qui sécrète l'exotisme balise un mode $\mathrm{d}^{\prime}$ approche de l'altérité qui préserve et conforte l'identité de celui qui regarde. Qualifier des êtres ou des choses d'exotiques, c'est signifier leur singularité, c'est-à-dire leur déviance par rapport à une norme conçue comme absolue et conforme à la vérité et à la réalité. Souligner l'étrangeté de l'Autre, c'est marquer en quelque sorte son extra-territorialité épistémologique. Loin de reconnaître la différence pour ce qu'elle est, c'est-à-dire l'essence même de l'Autre, l'exotisme confirme le même en conférant à celui qui définit l'étrangeté de l'Autre l'assurance de représenter la normalité. Le regard de celui qui attribue une valeur exotique à ce qui est étranger repose sur sa certitude de détenir la vérité. $C^{\prime}$ 'est à partir de celle-ci qu'il juge singulières, exotiques les moeurs de $l^{\prime}$ Autre $^{52}$. L'exotisme a précisément pour première fonction de fonder cette assurance.

Aussi le regard qui confère à l'Autre une dimension exotique ne peut-il être que valorisant. L'orgueil, voire la vanité marquent les échanges avec l'Autre. Il ne s'agit pas seulement de l'orgueil qui pousse, par exemple, le voyageur à étaler complaisamment ses aventures ou à manifester de la condescendance à l'endroit de ceux qu'il rencontre ${ }^{53}$,

50 L. P., p. 58. Dans son "Introduction", pour maintenir la fiction de son rôle de traducteur, Montesquieu écrit que les Persans, qui logeaient chez lui, le «regardaient comme un homme d'un autre monde» (L. P., p. 7).

51 On ne peut s'empêcher d'observer que l'exotisme naît au moment où l'Europe s'est imposée au monde et que l'Autre ne représente plus une menace.

52 Comme l'observe Victor Segalen, il est vrai sur un autre plan, «ne peuvent sentir la Différence que ceux qui possèdent une Individualité» (op. cit., p. 24). En même temps, sentir la différence, c'est aussi assurer son individualité: «la nature n'exista qu'au moment où l'homme sut la concevoir différente de lui» (ibid., p. 25, c'est l'auteur qui souligne). On peut inverser la proposition: l'homme n'eut conscience d'exister qu'au moment où il se perçut différent de la nature.

53 Eugène Fromentin: «il y a un plaisir irrésistible à dire d'un pays que peu de gens ont visité: "Je l'ai vu."» (op. cit., p. 186). Sur cette question, voir Régis Antoine, «La relation exotique», dans Revue des sciences humaines, juillet-septembre 1972, Nouvelle Série, fasc. 147, p. 376. 
mais de l'orgueil lié à la certitude de posséder la véritéà partir de laquelle on peut juger les moeurs de l'Autre. Regarder les coutumes de l'Autre en leur conférant un statut exotique, c'est se placer au-dessus de celles-ci pour les qualifier. La confrontation avec l'Autre confirme les valeurs à partir desquelles on mesure la distance que celui-ci entretient avec celles-ci et assure la supériorité de celui qui juge.

Ce va-et-vient entre l'Autre et soi, qui conforte le locuteur dans ses convictions, Usbek et Rica n'ont de cesse de le réaliser. Que le regard qu'ils portent sur l'Occident repose sur l'assurance de posséder la vérité, de nombreux textes dans le roman le suggèrent. Non seulement les deux voyageurs emportent-ils leurs croyances dans leurs bagages, mais en plus les autorités religieuses qu'ils consultent à l'occasion confirment les dogmes sur lesquels se fondent leur foi et leurs moeurs. En témoignent encore les lettres du premier eunuque du sérail d'Usbek, qui ne cesse d'avancer des axiomes que les événements ne parviennent pas à mettre en doute. Gardien du sérail et de l'orthodoxie, il rappelle à Usbek ses devoirs en même temps que sa foi.

Mais le confrontation avec l'Autre n'est jamais sans risques. Elle ramène à soi. La "réversibilité», pour emprunter un concept à Francis Affergan, constitue «un marqueur symbolique important de la démarche exotique ${ }^{54} \gg$. Le regard qui confère à l'Autre une dimension exotique est un regard décalé qui institue un retour à soi. Rica ne reconnaît-il pas que «nous jugeons jamais des choses que par un retour secret que nous faisons sur nous-mêmes ${ }^{55}$ » ? En qualifiant $l^{\prime}$ Autre d'exotique, le regard se place au centre des normes qui lui permettent de souligner sa différence. Non seulement se montre-t-il en train de se positionner au coeur de la norme, mais en plus il donne à voir les normes à partir desquelles il juge l'altérité. Le regard qui exotise, on me pardonnera ce néologisme, met à nue sa mécanique en révélant les modalités de la construction qu'il opère.

54 F. Affergan, Exotisme et altérité. Essai sur les fondements d'une critique de l'anthropologie, Paris, PUF, 1987, p. 105. Toutefois, son analyse s'inscrit dans la nostalgie de l'origine: il n'y a «de découverte de l'altérité qu'à travers une prescience nostalgique et un profond désir d'être troublé et de retrouver ainsi ses propres origines» (ibid., p. 105). $C^{\prime}$ est pourquoi il voit dans le Sauvage ce qu'il a été (op. cit., p. 105). Les deux autres «marqueurs symboliques» recensés par F. Affergan sont «le noeud nouveauté / identité où l'une ne peut advenir que sur fond de l'autre. Puis la soudaineté [où] l'abrupte dénivellation du premier instant de découverte n'a de sens qu'à partir d'une temporalité de l'attente et de l'attention» (ibid., p. 105). 
Cette mécanique, les Lettres persanes l'illustrent on ne peut mieux. En même temps que le voyage d'Usbek et de Rica donne à voir la réalité européenne sous un angle insolite, du moins par rapport aux valeurs musulmanes, il est l'occasion d'un retour sur celles-ci, comme le montrent les échanges entre Usbek et le mollah Méhémet-Hali. Plus grave, en même temps que les Persans font la critique de la société occidentale, ils assistent à la décomposition de leur propre univers, dont témoigne la révolte des femmes du sérail d'Usbek. Simultanément, si la société européenne forme un miroir dans lequel se mirent Usbek et Rica, ceux-ci retournent le miroir sur elle, ou plutôt contre elle. Une scène, en particulier, illustre ce jeu spéculaire. "Comment peut-on être Persan ?», se demandent des Parisiens qui trouvent Rica «bien extraordinaire $e^{56} »$. Mais dans le «bourdonnement» de la compagnie dans laquelle se trouve Rica bruit une autre question: «Comment peut-on être Français ?» Comment peut-on être si incapable d'ouverture à la différence ? L'étrangeté des Persans aux yeux des Français renvoie à leur propre étrangeté aux yeux des Persans, partant, à ceux des lecteurs. Au moment où le regard des Français transforme Rica en être exotique, le regard de ce dernier métamorphose les Français en objets étranges. Le regard qui souligne la différence de l'Autre met à nu sa propre singularité.

Une figure de rhétorique exprime ce double mouvement d'échange entre le même et le différent: le chiasme. L'exotisme repose essentiellement sur un dispositif de retournement ${ }^{57}$. Si la différence existe par le même, le même n'existe que par la différence, comme en témoigne exemplairement une scène du roman:

Les habitants de Paris sont d'une curiosité qui va jusqu'à l'extravagance [écrit Rica]. Lorsque j'arrivai, je fus regardé comme si j'avais été envoyé du Ciel: vieillards, hommes, femmes, enfants, tous voulaient me voir. Si je sortais, tout le monde se mettait aux fenêtres; si j'étais aux Tuileries, je voyais aussitôt un cercle se former autour de moi: les femmes mêmes faisaient un arc-en-ciel, nuancé de mille couleurs, qui m'entourait; si j'étais aux spectacles, je trouvais

56 L. P., p. 69.

57 Concept emprunté à Francis Affergan, pour qui «la vue se fait regard à la faveur d'un chiasme, d'un dispositif de croisement et d'une stratégie de retournement. Il y a regard quand il est permis que l'Autre me change et me fasse accéder à des recoins jusqu'alors cachés de ma propre identité» (op. cit., p. 155). Mais pour lui, ce dispositif permet essentiellement d'accéder aux recoins obscurs de l'être et de «retrouver ainsi ses propres origines» (ibid., p. 105). 
d'abord cent lorgnettes dressées contre ma figure: enfin jamais homme n'a tant été vu que moi ${ }^{58}$.

Le cercle de ceux qui regardent Rica forme aux yeux du Persan un arc-en-ciel aux mille teintes. Celui qui, par sa seule présence, devient spectacle aux yeux des Parisiens, retourne le regard que ceux-ci portent sur lui et transforme les Français en objet d'une représentation. En même temps qu'il est l'objet du regard d'autrui, Rica fait de ce regard l'objet du sien et il n'existe que dans la tension de ces regards croisés. Dès qu'il endosse un habit européen, sa physionomie cesse d'être «admirable» et il entre «tout à coup dans un néant affreux ${ }^{59}$ ». Voir et être vu constituent les deux faces d'une opération complémentaire qui définit certes l'essence de l'exotisme, mais aussi la raison d'être du voyageur, la condition même de son existence.

En regardant, Usbek et Rica s'exposent, aux deux sens du verbe, de s'afficher et de se mettre en péril. C'est qu'en même temps qu'ils découvrent, c'est-à-dire, dévoilent la différence de l'autre, son exotisme, ils manifestent son irréductibilité, sa résistance essentielle à ce qu'ils sont. Du coup, pour éviter d'entrer dans le néant, pour être, Usbek et Rica doivent manifester à leur tour leur différence. Mais pour être différents, ils doivent constater celle de ceux qui les regardent, car c'est à partir de celle-ci qu'ils sont différents et qu'ils existent. L'exotisme, écrit Victor Segalen, «n'est donc pas une adaptation; n'est donc pas la compréhension parfaite d'un hors soi-même qu'on étreindrait en soi, mais la perception aiguë et immédiate d'une incompréhensibilité éternelle ${ }^{60}{ }^{\prime}$, $c^{\prime}$ est-à-dire la perception aigüe de l'être-là de l'Autre ${ }^{61}$. L'Autre devient le miroir réfléchissant qui assure de son existence celui qui le regarde ${ }^{62}$.

58 L.P., p. 68.

59 L.P., p. 69.

60 V. Segalen, op. cit., p. 25. Segalen parle alors «d'impénétrabilité» (p. 25). Pour sa part, F. Affergan écrit que «l'Autre est reconnu en tant qu'il est porteur d'un ailleurs irréductible et toujours atopique» (op. cit., p. 104).

61 Pour Tzvetan Todorov, le «dialogue des cultures" implique "une affirmation de l'extériorité de l'autre qui va de pair avec sa reconnaissance en tant que sujet» ( $L a$ Conquête de l'Amérique, Paris, Seuil, 1982, p. 254), mais ici, c'est un rapport dialectique entre la nécessaire reconnaissance de l'autre comme sujet et les risques de celle-ci qui est mis en relief.

62 On peut penser ici à la rencontre d'Aziyadé: «Aziyadé me regardait fixement», écrit Pierre Loti. «Devant un Turc, elle se fût cachée; mais un giaour n'est pas un homme; tout au plus est-ce un objet de curiosité qu'on peut contempler à loisir» (op. cit., p. 36). 
Pour être, Usbek et Rica doivent reconnaître aux Français le statut de sujet du regard à travers lequel ils existent, au moment même où ils les transforment en objet de leur propre regard ${ }^{63}$.

Cette dialectique, ce hors champ, dans lequel se constitue la double conscience d'être et d'être par autrui, qui transforme Usbek et Rica en objets du regard d'autrui au moment même où ils transforment celui-ci en objet de leur regard, constitue une position ontologique intenable. Heureusement, l'exotisme distille son propre antidote. On connaît l'importance de l'ironie dans le discours philosophique. Arme redoutable, en ce qu'elle raille les positions adverses tout en semblant soutenir le contraire de ce qu'elle affirme, l'ironie ne laisse pas même la chance à sa victime, qu'elle déporte en dehors de la raison, de se défendre. Le regard de l'observateur qui nappe d'exotisme ce qui est différent de lui est lui aussi, à sa façon, porteur d'ironie, l'étonnement qu'il implique soulignant le caractère phénoménal et excentrique de ce qu'il regarde. En plus, le regard qui exotise, parce qu'il signale un écart sous une apparente bénévolence, interdit toute réplique. Qui mieux est, la distance qu'il maintient entre lui et $l^{\prime}$ Autre le protège ${ }^{64}$.

Le paradoxe d'un roman exotique sans exotisme n'est qu'apparent. Dans les Lettres persanes sont mises en place les structures de l'exotisme. Ce roman, qui exploite si peu l'exotisme, en illustre les modalités, l'usage, finalement le sens. Dans l'exotisme, dans le chassé-croisé des regards des uns et des autres, se joue l'existence des êtres. L'exotisme n'est pas l'occasion d'une reconnaissance de l'Autre, mais l'espace d'un conflit qui met en jeu l'existence même des êtres. C'est cet enjeu qu'illustrent

Ce n'est que dans la reconnaissance d'Aziyadé que le narrateur existera. Toute la passion amoureuse créée par le narrateur ne vise, peut-être, qu'à assurer son existence.

63 Dans ses «Réflexions sur les Lettres persanes», Tzvetan Todorov observe: «la connaissance de soi est possible mais elle implique au préalable celle des autres» (Romanic Review, mai 1983, vol. LXXIV, n³, p. 308).

64 Sans cesse dans les Lettres persanes affleure l'ahurissement d'Usbek devant des coutumes des Français, dont ses observations suggèrent le caractère absurde, sans cesse affleure aussi l'ironie de Montesquieu qui met en scène un voyageur qui, au moment même où il critique les moeurs européennes, assiste à l'effondrement de son propre univers. Sur l'ironie dans les Lettres persanes, voir Pierre Cambou, «L'Esprit et la loi dans les Lettres persanes: une pratique ironique des Lumières", Littératures, printemps $2000, n^{\circ} 42$, p. 37-51. Voir notamment l'analyse de la scène au cours de laquelle des Français demandent à Rica comment on peut être persan (p. 39). 
magistralement les Lettres persanes grâce à un dispositif narratif tout en trompe-l'oeil. Penseur lucide qui réalise pleinement le projet des Lumières, Montesquieu démonte un phénomène au moment même où il est en train de naître et en dégage le caractère instrumental pour annihiler l'Autre. Peut-on faire un meilleur usage de l'exotisme que d'en exposer la perversion?

\section{Annexe}

À la suite d'Oscar Bloch et de Walther von Wartburg (Dictionnaire étymologique de la langue française, Paris, PUF, 1960), Le Grand Larousse de la langue française (Paris, Librairie Larousse, 1971, vol. 3, p. 1823) et Le Grand Robert de la langue française (deuxième édition dirigée par Alain Rey, Paris, Dictionnaires Le Robert, 2001, vol. 3, p. 444), indiquent erronément que le substantif est signalé dans le Dictionnaire national ou Dictionnaire universel de la langue française, de Louis-Nicolas Bescherelle, où seul l'adjectif «exotique» est recensé (Paris, Simon et Garnier, 1845). De fait, dans la première édition du Dictionnaire étymologique de la langue française (Paris, PUF, 1932), O. Bloch et W. Von Wartburg indiquaient la date de 1866, sans doute à la suite d'Émile Littré, qui, dans son Supplément (Paris, Librairie Hachette et $C^{\text {ie }}, 1878$, p. 151), notait la présence du substantif dans les Mélanges tirés d'une petite bibliothèque romantique, de Charles Asselineau (Paris, Chez René Pincebourde, Éditeur, 1866, p. 122). Ce n'est qu'à partir de la troisième édition du Dictionnaire étymologique de la langue française (1960) que W. von Wartburg retient, par erreur, la date de 1845. Dans son Dictionnaire étymologique de la langue française (Paris, Librairie Larousse, 1938), Albert Dauzat indiquait la date de 1866; dans celle de 1964, sous la direction d'A. Dauzat, de Jean Dubois et de Henri Mitterand, la date retenue est 1845 (Paris, Librairie Larousse, 1964). Pour leur part, les rédacteurs du Trésor de la langue française (Paris, Éditions du C. N. R. S., 1980, t. 8, p. 460), signalent que les frères Goncourt emploient le substantif dans leur Journal, le dimanche 20 février 1860, alors qu'ils observent au sujet de Gustave Flaubert: «trois femmes en peignoir de soie, filant du dos au talons (sic), flanquées d'un négrillon habillé de nanquin et chaussé de babouches: un monde qu'il entrevoyait dans un patio tout plein de fleurs des tropiques, et chantait au milieu un jet d'eau, - pour ce jeune Normand qui n'avait encore voyagé que de Normandie en Champagne et de Champagne en Normandie, c'était d'un exotisme bien tentant» (Journal, texte intégral établi et annoté par Robert Ricatte, Monaco, Les Éditions de l'Imprimerie nationale de Monaco, 1956, t. III, p. 227). 